\title{
Interroger les nouvelles politiques d'éducation et de formation en France depuis 25 ans : une recomposition parallèle des conceptions de la justice et des formes de l'État Question the New Education and Further Training Models Implemented in France over the Last 25 Years : A Parallel Recomposition of the Conception of What is Right and State Forms
}

\section{Jean-Louis Derouet}

Volume 40, numéro 1, printemps 2008

Les nouvelles politiques d'éducation et de formation

New Policies in Education and Training

URI : https://id.erudit.org/iderudit/019472ar

DOI : https://doi.org/10.7202/019472ar

Aller au sommaire du numéro

\section{Éditeur(s)}

Les Presses de l'Université de Montréal

\section{ISSN}

0038-030X (imprimé)

1492-1375 (numérique)

\section{Découvrir la revue}

Citer cet article

Derouet, J.-L. (2008). Interroger les nouvelles politiques d'éducation et de formation en France depuis 25 ans : une recomposition parallèle des conceptions de la justice et des formes de l'État. Sociologie et sociétés, 40(1), 57-68. https://doi.org/10.7202/019472ar

\section{Résumé de l'article}

Le but de l'article est de déplacer les interrogations sur la crise du modèle de démocratisation en place depuis la fin de la Deuxième Guerre mondiale. Ce modèle est fondé sur une séparation de l'école et de la société. Ce qui était prôné comme une protection devient une perte de sens : les élèves d'origine populaire ne retrouvent pas leurs enjeux dans les « épreuves en papier » de l'école. Cette idée est illustrée par une analyse de la situation française et en particulier des résultats de la politique visant à amener $80 \%$ d'une génération au niveau du baccalauréat. La croissance des résultats a été spectaculaire de 1985 à 1995. Depuis, la courbe flotte et tend même à décroître. Cette crise peut être interprétée dans un cadre plus large : le déclin de l'État-providence et la montée de l'État managérial. Celui-ci relève d'un nouveau modèle de démocratisation : plus de séparation entre le temps des études et le temps de la production, mais une formation tout au long de la vie ; une gouvernance en réseau qui fait une large place aux intérêts économiques... Paradoxalement, ce nouveau modèle qui correspond à la nouvelle organisation du capitalisme pourrait peut-être répondre plus adéquatement au désengagement des classes populaires que l'ancienne forme scolaire.
Tous droits réservés @ Les Presses de l’Université de Montréal, 2008
Ce document est protégé par la loi sur le droit d'auteur. L’utilisation des services d'Érudit (y compris la reproduction) est assujettie à sa politique d'utilisation que vous pouvez consulter en ligne.

https://apropos.erudit.org/fr/usagers/politique-dutilisation/ 


\section{Interroger les nouvelles politiques d'éducation et de formation en France depuis 25 ans: une recomposition parallèle des conceptions de la justice et des formes de l'État}

JEAN-LOUIS DEROUET

Unité mixte de recherche Éducation et Politiques

Institut national de recherche pédagogique

Université Lyon 2

19, allée de Fontenay, B.P. 17424

69347 Lyon cedex 07, France

Courriel: jean-louis.derouet@inrp.fr

$\mathrm{D}$

E NOMBREUX TRAVAUX ONT MIS EN ÉVIDENCE les liens qui existent entre les nouvelles politiques d'éducation et de formation et le nouvel esprit du capitalisme (Boltanski, Chiapello, 1999): omniprésence de la philosophie du projet, obligation de résultats, organisation réticulaire du monde, etc. De même, la formation tout au long de la vie accompagne la flexibilité et la mobilité correspondant au nouvel ordre économique... Sans nullement remettre en cause ces analyses et leurs fondements épistémologiques ou politiques, le présent article voudrait déplacer le regard et montrer que cette évolution correspond aussi à un épuisement du modèle de démocratisation porté par la gauche pédagogique depuis la fin de la Deuxième Guerre mondiale. Sa conception repose sur une «forme scolaire» (Vincent, 1980) qui sépare le temps des études de celui de la production et prône l'allongement du temps des études pour tous. Cette organisation a toujours mis mal à l'aise une partie des enfants d'origine populaire. Ce phénomène prend de l'ampleur et aboutit aujourd'hui, dans certains établissements, à une sorte d'exit généralisé (Hirschman, 1970). Le fonctionnement en réseau proposé par la nouvelle organisation du capitalisme qui permet des rapprochements entre les écoles et les entreprises répond à une vraie question, même si on peut considérer qu'elle en détourne le sens. 
La démonstration s'appuie sur une analyse empirique concernant la France. En 1984, l'objectif d' «amener $80 \%$ d'une génération au niveau du baccalauréat » constituait l'accomplissement du modèle traditionnel. Après 10 ans de succès, celui-ci a visiblement perdu son pouvoir mobilisateur. Pourquoi ce modèle, qui a permis dans les années 1960 et 1970 aux enfants des classes moyennes d'accéder à l'école de la bourgeoisie, ne convient-il plus aujourd'hui aux enfants des nouvelles classes populaires? Les deux premières parties de l'article tentent de répondre à cette question. Une troisième partie envisage les déplacements théoriques qu'entraîne cette analyse. Elle dégage une recomposition parallèle des formes de la justice et des formes de l'État. L'ancienne définition de la justice reposait sur un «grand renfermement». La séparation de l'école du reste de la société était censée assurer l'indépendance des jugements scolaires et cette indépendance était garantie par l'État. Celui-ci utilisait en même temps l'école pour assurer une mission de partage des bénéfices (Darras, 1966) et de compensation des inégalités sociales. Aujourd'hui, la nouvelle conception suppose une intégration de la formation dans la production. À l'extrême, plus de système de formation séparé mais des entreprises apprenantes. De cette façon, le secteur de la formation est soumis à la même référence de justice que l'ensemble de la société: l'obligation de rendre compte. Si les classes populaires peuvent retrouver dans cette proposition quelques-uns de leurs intérêts, la convergence est pleine d'ambiguïtés. Nous tenterons dans la conclusion de les analyser et de réfléchir à la manière de construire un nouveau projet de démocratisation des études.

\section{LA «RUPTURE DE 1995 » EN FRANCE : EXIT DES CLASSES POPULAIRES PAR RAPPORT AU PROJET DÉMOCRATIQUE TRADITIONNEL?}

Jusqu'au milieu des années 1990, la France s'est inscrite dans le modèle traditionnel de démocratisation, dont un objectif est l'allongement du temps de formation. Cette définition du bien commun repose sur une conception élaborée à l'époque moderne et qui s'est affirmée à la fin du XVIII ${ }^{\mathrm{e}}$ siècle (Backzo, 1982). Le principe est de créer un espace intermédiaire où les jeunes peuvent s'exercer à l'abri des contraintes de la vraie vie. À partir du moment où cette séparation a été admise par la société, l'idée s'est imposée que le progrès, pour les individus comme pour l'humanité, reposait sur l'allongement du bienheureux «temps des études» (Verret, 1975). Cet objectif a inspiré l'ensemble des politiques scolaires du $\mathrm{xx}^{\mathrm{e}}$ siècle. Tout d'abord, une longue marche vers un collège unique qui rassemble tous les élèves jusqu'à 15 ou 16 ans. Après la loi de 1975, ce mouvement a été prolongé par le ministre Jean-Pierre Chevènement qui a proposé en 1984 d'amener $80 \%$ d'une génération au niveau du baccalauréat en l'an 2000. Ce slogan se situe dans le prolongement du rapport d'Antoine Prost (1983) qui relevait, 10 ans après la loi instaurant le collège unique, une demande croissante pour un prolongement de la scolarité au lycée. Il a cependant fait l'objet de nombreuses critiques. Beaucoup, à droite comme à gauche, craignaient un «bradage» du diplôme. La réussite du pari reposait sur un baccalauréat professionnel dont le statut était discuté: s'agirait-il d'un vrai baccalauréat ou d'un «brevet dopé»? Une partie de la gauche s'in- 
terrogeait aussi sur le sort des $20 \%$ qui n'atteindraient pas ce qui allait devenir le seuil de normalité. La société s'est néanmoins engagée avec enthousiasme dans la voie alors ouverte. Antoine Prost, qui avait identifié cette attente, avait également posé la question du sens de ce glissement: les élèves qui poursuivent leurs études sont-ils vraiment mus par un intérêt pour le savoir ou par un projet de mobilité sociale? Ne sont-ils pas plutôt prisonniers d'une organisation pour laquelle un jeune n'a plus d'autre place dans la société que celle d'étudiant?

Ce mouvement a été entériné par la loi d'orientation de 1989, posant comme principe que tous les jeunes devaient sortir du système éducatif avec une qualification: un $\mathrm{CAP}$ ou un $\mathrm{BEP}^{1}$ au moins; le niveau du baccalauréat pour $80 \%$ d'une génération. Elle a aussi mis en place une évaluation nationale de performance des élèves qui devait répondre aux inquiétudes concernant une éventuelle baisse de niveau. L'adhésion de la société s'est traduite par une massification des lycées et du premier cycle de l'enseignement supérieur. En 1995, tous les observateurs pensaient que la réussite du pari était à portée de main: de 31,2\% en 1986, la proportion de bacheliers dans une génération était passée à $62,7 \%$. C'est à cette date que la courbe a commencé à flotter: le pourcentage a été de $64,2 \%$ en 2006 . Non seulement la progression stagne, mais la part relative du baccalauréat général a diminué: de 37,2 \% d’une génération en 1995, elle est tombée à $35 \%$ en $2006(17,2 \%$ pour les bacs technologiques et 12,3\% pour les bacs professionnels).

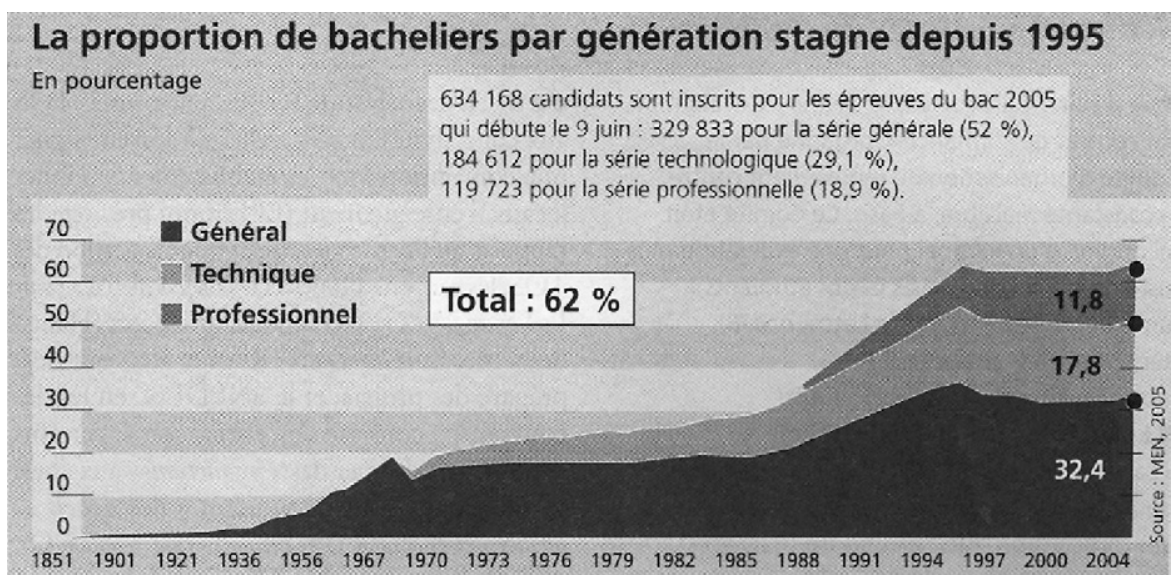

La perception de cette cassure est brouillée par la confusion avec le taux de réussite à l'examen, qui était effectivement de $83,3 \%$ en 2007. Ce taux ne dit rien évidemment sur le pourcentage que les candidats représentent au sein de leur génération.

1. CAP : certificat d'aptitude professionnelle; BEP : brevet d'études professionnelles. Ce sont des diplômes d'études secondaires en enseignement professionnel. 


\section{LES CHIFFRES}

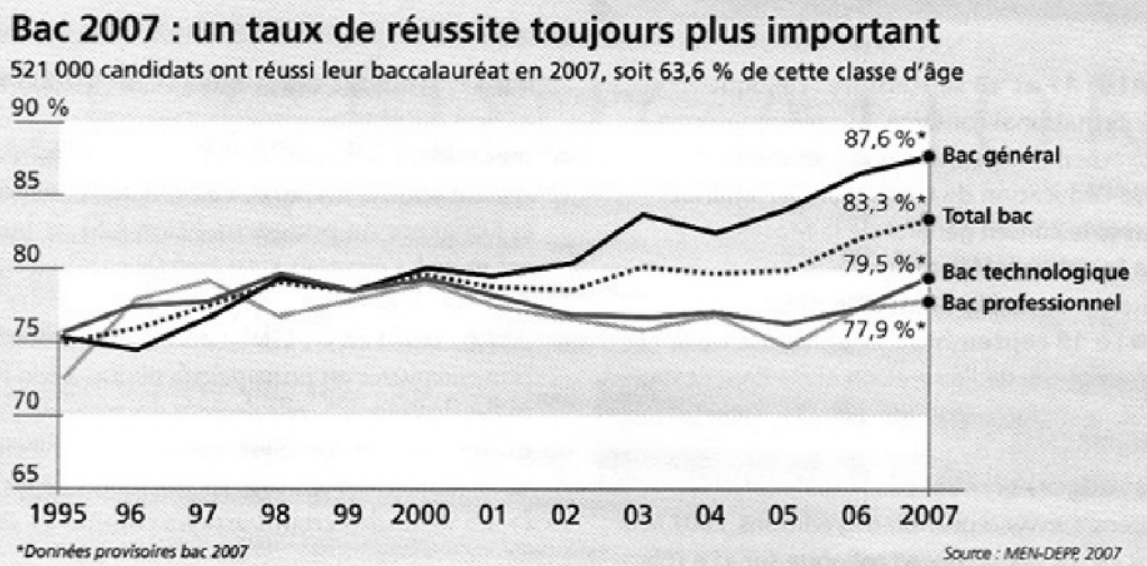

Le phénomène doit être analysé avec prudence. Tout d'abord, le slogan de JeanPierre Chevènement portait sur le niveau du baccalauréat, non pas sur la réussite à l'examen. La rupture est néanmoins significative. Ensuite, d'autres indications montrent que le niveau de scolarisation continue à progresser. L'OCDE (2004) constate que dans tous les pays y compris la France, la proportion des élèves qui s'arrêtent avant la fin du second cycle a baissé entre 1991 et 2002 et la proportion des 25-34 ans détenant un diplôme du supérieur tertiaire a augmenté. Même si elles ne sont pas totalement incompatibles, ces données sont trop variées pour en tirer de suite des conclusions. Surtout, l'analyse se trouve vite confrontée à des débats impossibles à trancher. Ainsi de celui qui porte sur l'interprétation du phénomène de massification. La massification n'est pas la démocratisation, mais y contribue-t-elle? Les données disponibles nourrissent l'une et l'autre thèse et il faut remonter assez loin dans les épistémologies qui les ont produites pour en comprendre les différences (Merle, 2002). Il ne s'agit là que d'un exemple. La théorie du capital humain suppose un lien direct entre la diffusion des savoirs dans la société et la performance économique. Ce lien est depuis longtemps mis en question (Duru-Bellat, 2006). Bien plus, il n'est pas sûr que l'accroissement du niveau de diplôme ait des effets significatifs sur la diffusion des idéaux démocratiques (Baudelot, Leclerq, 2004). Un débat du même ordre peut s'engager sur un malaise des enfants d'origine populaire vis-à-vis de la forme scolaire. Le phénomène est identifié depuis longtemps. C’est même le cœur de la théorie de la résistance proposé par Paul Willis (1981). L'attention portée aujourd'hui aux manifestations d'indiscipline et de violence correspond-elle à un accroissement réel du phénomène ou à changement de regard? Les résultats des enquêtes récentes laissent sur ce point une impression mitigée (Charlot, 2001).

Au-delà de ces différentes interprétations, l'essentiel est peut-être un désenchantement social qui dépasse les accords ou les désaccords entre analyses scientifiques. Les défauts dénoncés aujourd'hui ne sont pas nouveaux, mais ils ont été longtemps vus comme les faiblesses d'un fonctionnement globalement positif et qu'il était possible 
d'améliorer. Ils sont plutôt vus aujourd'hui comme les symptômes d'un échec de ce système, qui invitent à revenir sur de dangereuses illusions. Le cas du collège unique concentre toutes ces interrogations: combien de temps les enseignants resteront-ils attachés à un idéal d'égalité des chances qui ne sert qu'à les rendre malheureux et à les désigner comme boucs émissaires (Dubet, Duru-Bellat, 2002)?

Quelques spécialistes de sciences de l'éducation commencent à s'interroger sur le sens de l'allongement sans fin des études (Duru-Bellat, 2006). Ces initiatives sont importantes, car la discipline s'est le plus souvent contentée d'accompagner l'expansion du projet de démocratisation dans son sens traditionnel.

Même si la crise reste mal identifiée et mal expliquée, les organisations internationales ont proposé une alternative qui a été reprise en France par la loi d'orientation de 2005. Celle-ci prolonge le compromis de la loi de 1989: une qualification pour tous, le niveau du baccalauréat pour $80 \%$ d'une génération. Cette orientation a même été renforcée, après l'élection de Nicolas Sarkozy en 2007, par un plan qui propose d'amener $60 \%$ d'une génération au niveau de la licence. Toutefois, ce volontarisme n'est pas incompatible avec une nouvelle manière de découper le processus de formation. L'État garantit à tous les jeunes l'acquisition d'un socle commun de connaissances et de compétences vers 15 ou 16 ans. Ensuite, chacun construit son parcours dans un univers réticulaire qui relie formation et production. Certains suivent la voie classique: le lycée, avec parfois des adaptations (une année de seconde en deux ans, une progression par unité de valeurs ${ }^{2}$, etc.), le baccalauréat et l'enseignement supérieur. D’autres empruntent des voies différentes: formation professionnelle en alternance, reprise des études au cours de la vie professionnelle, acquisition de diplômes par validation des acquis de l'expérience, etc. Cette conception est illustrée par la nouvelle répartition des départements ministériels. Une hésitation était perceptible depuis plusieurs années: faut-il rassembler dans un même ministère tout ce qui concerne la formation et l'enseignement: enseignement scolaire, université, voire culture? Ou faut-il séparer la formation pour tous de celle qui concerne l'élite et constituer un ministère séparé pour les universités et la recherche? Nicolas Sarkozy a effectué un partage clair. Non seulement le ministère des Universités est séparé de celui de l'Éducation nationale, mais la loi de juillet 2007 sur l'autonomie des universités impose à ces institutions le fonctionnement réticulaire caractéristique de la nouvelle organisation du capitalisme. En revanche, le ministère de l'Éducation nationale, qui devient le ministère de l'Éducation scolaire, est confié à un militant gaulliste attaché au pouvoir régulateur de l'État.

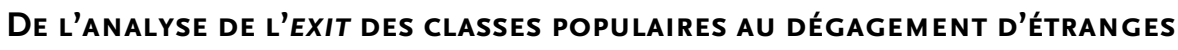 CONVERGENCES: LA FIN DU «GRAND RENFERMEMENT» DE L'ÉDUCATION}

$\mathrm{Si}$ ce nouveau modèle se met en place, c'est en partie grâce à sa capacité à récupérer la critique de l'école émise par les forces de gauche dans les années 1960 et 1970: dénonciation de la bureaucratie et du corporatisme, mise en cause d'une "coupure entre

2. C'est-à-dire par crédits. 
l'école et la vie», «indifférence aux différences» qui ignore les personnes et transforme l'égalité de traitement en mystification. Ces critiques, élaborées dans une perspective de progrès, nourrissent aujourd'hui l'argumentaire du capitalisme managérial. Cette récupération est à l'origine d'une crise de la critique qui a déjà été analysée. Il est ainsi difficile de rappeler la mission de l'État concernant l'encadrement du libre jeu de la concurrence sans passer pour un jacobin attardé, voire un nostalgique de la planification soviétique (van Haecht, 2004). Il serait toutefois injuste de limiter l'explication des progrès de la nouvelle organisation à la faiblesse et aux contradictions des oppositions. Celle-ci tient aussi par sa capacité à récupérer le malaise des classes populaires devant la forme scolaire. L'analyse retrouve, dans un autre contexte, une nouvelle manifestation de cette "étrange convergence» que Jean-Claude Chamboredon avait mise en évidence à propos de la «surprenante et très compréhensive rencontre entre Foucault et Ariès, devenus dans les années 1970 le Janus bifrons de la critique du système d'enseignement» (1988). La critique de la violence exercée par les institutions d'éducation, d'inspiration gauchiste ou libertaire, rejoignait les critiques libérales plus anciennes: celles de l'étatisme, du Moloch de la société bureaucratique, etc.

Plusieurs causes ont été envisagées pour expliquer la rupture de 1995: un changement d'organisation du baccalauréat; une anticipation rationnelle des chances de réussite des jeunes d'origine populaire dans une société marquée par le chômage et la déqualification. L'essentiel paraît ailleurs. Dès le collège, les enfants d'origine populaire se retirent du jeu. Les résultats d'une enquête menée par Robert Ballion peuvent aider à comprendre les raisons de ce désengagement (1989). Il a interrogé les nouveaux lycéens participant du projet de conduire $80 \%$ d'une génération au niveau du baccalauréat. Ces jeunes doivent souvent travailler pour payer leurs études. Ils sont sensibles au facteur d'inégalité que constitue cette contrainte. En même temps, ils affirment que ce contact avec la «vraie vie» les aide à donner du sens aux «épreuves en papier» de l'école.

L'analyse de cette nouvelle donne nécessite un retour sur l'histoire de la séparation entre formation et production. L'idée d'un temps des études séparé du reste de la vie sociale est récente. Les historiens discutent les théories d'Ariès (1975) : le sentiment de l'enfance a certainement existé avant l'époque moderne, mais il est certain que l'Ancien Régime a cultivé un modèle de compagnonnage où les jeunes se formaient en participant à l'activité des adultes. Ce modèle assurait la transmission des valeurs en même temps que celle des savoirs et des savoir-faire. Il présentait par là même le risque de restreindre les enfants à leur condition sociale, voire de les soumettre aux préjugés dont leur milieu faisait l'objet. C'est contre cette reproduction qui correspondait à une société d'ordres que s'est construit le projet des Lumières (Backzo, 1982). Celui-ci établit une séparation des temps, des pouvoirs et des savoirs. L'exterritorialité de l'école est censée garantir l'indépendance des jugements scolaires. Surtout, l'école sélectionne parmi les savoirs sociaux ceux qui correspondent à son projet, c'est-à-dire ceux qui initient les enfants à sa conception de l'intérêt général. Elle met ces savoirs en forme pour constituer des disciplines, met au point une batterie d'exercices canoniques, élabore un système d'évaluation, etc. 
Cette conception est à l'origine d'incontestables progrès et elle garde une position très forte dans le débat politique: l'école porte les valeurs du monde qui doit être, et il faut la protéger des pressions du monde qui est (Alain, 1932; Finkielkraut, 1989). Différentes indications laissent cependant penser qu'un cycle s'achève. La séparation avait été conçue à l'origine comme une protection des enfants contre la rudesse des épreuves sociales. Elle n'est plus comprise comme telle. Elle apparaît comme une coupure arbitraire qui crée un monde artificiel, réservé aux «forts en thème» et dissocie les études de leurs enjeux sociaux pour la majorité des élèves.

\section{L'ÉMERGENCE DE L'ÉTAT MANAGÉRIAL}

À l'issue de cette analyse, il est possible d'avancer une hypothèse interprétative qui situe l'épuisement relatif d'un modèle de démocratisation et la proposition d'un autre modèle à l'intérieur d'un cadre plus large. Celui-ci ne se limite pas au domaine de l'éducation. Il concerne tous les secteurs d'intervention traditionnels de l'État-providence: santé, éducation, droit du travail et protection sociale. Partout, on assiste à une recomposition parallèle des formes de la justice et des formes de l'État, dont le prototype est la proposition de Troisième Voie britannique (Giddens, 1988)

L'évolution remonte au rapport A Nation at Risk, paru aux États-Unis en 1983. Celui-ci a jeté les bases d'un nouveau système de référence selon lequel le but essentiel de l'éducation et de la formation n'est plus l'égalité ou la cohésion à l'intérieur d'une société, mais le maintien du rang du pays face à la concurrence mondiale. Ce déplacement des objectifs amène une reformulation de la tension traditionnelle entre projet d'égalité et exigence d'efficacité. La conception de la justice en éducation a toujours dû tenir compte de la division du travail et intégrer l'obligation de performance. Toutefois, il s'est longtemps agi d'une contrainte extérieure avec laquelle il fallait négocier un compromis. C'est le cas du modèle de l'égalité des chances. Entre un idéal inaccessible d'égalité des conditions et la dure réalité de la production, il introduit une sélection par le mérite. La nouvelle conception considère l'obligation de résultat comme partie intégrante de sa morale. C'est un aspect de l'obligation de reddition de compte: rendre compte à l'État et au contribuable de l'argent dépensé, rendre compte aux usagers de la qualité des services qui leur sont proposés, etc.

Cette nouvelle définition de la justice a plusieurs conséquences sur le projet de formation et sa mise en œuvre. Elle réhabilite tout d'abord l'intérêt pour la formation des élites. C'est de leur dynamisme que dépendent les performances de l'ensemble de la société et celles-ci profitent à tous. En même temps, la définition de ces élites évolue. Il ne s'agit plus d'assurer la transmission de la culture gréco-latine mais de s'insérer précocement dans des réseaux internationaux. Cela implique non seulement la maîtrise de l'anglais, mais aussi une philosophie fondée sur le projet et la mobilité.

L'idéal démocratique d'élévation du niveau culturel pour tous n'est cependant pas abandonné. La formation de la main-d'œuvre aussi constitue un atout dans la compétition internationale. Le rapport A Nation at Risk prône en ce domaine un retour au réalisme. Une valorisation des dimensions culturelles a mis en difficulté les enfants 
d'origine populaire et jeté un trouble qui nuit à la qualité de leur formation. Il faut revenir aux fondamentaux (back to basis). Dans un contexte de concurrence internationale où la régulation repose sur le benchmarking, ces fondamentaux doivent être exprimés en termes de compétences et non en termes de savoirs toujours liés à des programmes nationaux. Cet ensemble de déplacements conduit à un nouveau phasage des études. L'État garantit à tous un socle commun de compétences acquises à la fin de la scolarité obligatoire. Ensuite, il appartient à chaque individu de construire son parcours de formation tout au long de la vie: le lycée et l'université pour certains; un retour en formation comme seconde chance à ceux qui n'ont pas réussi dans la période scolaire; une validation des acquis de l'expérience (VAE) sans passer par la forme scolaire, etc.

Ce modèle a peu à peu servi de référence à l'ensemble du monde. Il faut bien sûr se garder de toute vision mécaniste de la mondialisation (Dale, 2000), mais le contexte de concurrence a partout les mêmes effets. Les orientations portées par les nouvelles politiques américaines et la Troisième Voie britannique ont été généralisées par les recommandations de la Conférence de Lisbonne en 2000 et par la mise en place d'une Méthode ouverte de coordination (MOC). La Conférence a fixé un objectif: que l'Europe occupe en 2010 la première place dans une économie de la connaissance. Pour l'atteindre, il est nécessaire que les États membres rendent compte de leurs performances en matière de formation. Pour cela, ils doivent définir des standards de comparaison, ensuite utilisés pour mettre en place des dispositifs d'évaluation nationaux et une régulation par benchmarking: les États qui ont les résultats les plus faibles sont invités à s’inspirer des «bonnes pratiques» de ceux qui réussissent.

Cette évolution du programme de justice sous-tend une nouvelle conception de l'État que l'on peut qualifier d'État managérial parce que son principe de régulation est l'obligation de résultat (Gewirtz, 2008). Contrairement à ce qui est parfois déploré, il ne s'agit pas d'un déclin. L'État se désengage certes de certaines obligations antérieures: dans un contexte de concurrence mondiale, la pression fiscale entraîne la délocalisation des activités et donc des emplois. Pour contrer cette dérive, les États cherchent à transférer une partie de la dépense d'éducation vers les collectivités locales et les familles. En France, ce phénomène commence seulement. L'État reste le principal pourvoyeur de fonds puisqu'il assure le salaire des fonctionnaires, mais sa part a diminué de 69,5\% en 1980 à $63,6 \%$ en 2005, alors que celle des collectivités a augmenté de 14,2\% en 1980 à $21,2 \%$ en 2005 . L’université est le secteur le moins touché par cette évolution: la part de l'État reste de $77 \%$ contre $9 \%$ pour les familles et $6 \%$ pour les collectivités territoriales. Il faudra néanmoins être attentif aux conséquences de la loi de juillet 2007 sur l'autonomie des universités. Cette autonomie sera d'autant plus forte qu'elle pourra s'appuyer sur des financements complémentaires, publics ou privés. Ce désengagement ne constitue toutefois qu'un aspect des évolutions actuelles. L'obligation de résultat amène par ailleurs à mettre en place des dispositifs qui renforcent le pouvoir de l'État même s'ils déplacent les lieux de son exercice: les États de tradition centralisée passent du contrôle a priori à l'évaluation a posteriori. En France, la Loi organique sur la 
loi des finances (LOLF), votée en 2001 et mise en œuvre dans le domaine de l'éducation à partir de 2006, encadre l'autonomie accordée aux établissements par les récentes mesures de déconcentration et de décentralisation. Chaque secteur du service public doit expliciter ses objectifs et fournir des indicateurs de résultats.

\section{Conclusion}

L'allongement du temps des études ne constitue plus la définition unique du bien dans le domaine de l'éducation. L'accès au lycée et à l'université ne constitue qu'un des parcours possibles après le socle commun. Cette évolution amène à reprendre une des questions classiques de la philosophie politique de l'éducation: comment passer de façon juste de l'école pour tous à l'école pour les meilleurs? Et qui sont les meilleurs? Cette question devrait être au centre du débat public dans les années qui viennent. Il y a en effet pour le moment une tension entre les différentes recommandations de la Communauté européenne. Certaines se situent dans le prolongement des politiques de démocratisation des années 1960 et 1970. D’autres explorent les différentes voies proposées par le nouveau modèle: enseignement en alternance, formation tout au long de la vie, «entreprises apprenantes», etc. Une période de tuilage s'annonce. Chaque pays s'oriente à sa manière. Il faudra bien sûr suivre avec attention l'évolution des débats.

Un point essentiel sera l'évaluation des résultats des deux promesses de l'État managérial: la garantie d'un socle commun à 15 ou 16 ans, l'accompagnement des parcours individuels au sein de cet univers réticulaire ou alternent les périodes de formation et les périodes de production.

Sans revenir sur les querelles concernant le contenu du socle commun et ses rapports avec le projet de culture commune porté par la gauche pédagogique depuis le plan Langevin-Wallon, est-on bien sûr que ce socle sera vraiment commun? Ne risquet-on pas de retrouver ce taux d'échec de plus ou moins $20 \%$ identifié depuis 1975 ? Les premières études statistiques nationales sur la réussite scolaire avaient mis en évidence une régularité: plus ou moins $20 \%$ des élèves redoublent le cours préparatoire parce qu'ils ne savent pas lire, $20 \%$ des élèves sont orientés en fin de cinquième ${ }^{3}$ vers des formations courtes. Cette situation a peu évolué. On retrouve ce pourcentage d'échec dans l'objectif d'amener $80 \%$ d'une génération au niveau du baccalauréat. $\mathrm{Ne}$ risque-t-on pas de le retrouver dans l'atteinte des objectifs du socle commun? Et, dans ce cas, quelle est l'alternative? L'État peut-il offrir un vrai travail et une réelle possibilité de retourner en formation à des jeunes qui n'ont pas profité de cette première formation? Ou ceux-ci entreront-ils dans un cycle sans fin de précarité et d'assistanat? Dans ce socle commun même, comment se renégocie le rapport entre égalité et efficacité? L'exemple américain permet de cerner le problème. Une vingtaine d'années après le rapport A Nation at Risk, la loi No Child Left Behind (2002) a proposé un nouveau

3. Le cours préparatoire correspond aux deux premières années du primaire. La cinquième correspond à la deuxième année du secondaire. 
compromis. Le nouveau système ne s'intéresse pas seulement aux performances des élites, il vise aussi la réussite de tous. Les moyens sont d'abord ceux du libéralisme: concurrence entre les établissements, identification et dissémination de bonnes pratiques, incitations financières pour les enseignants qui réussissent, etc. Mais la remise à niveau des élèves en difficulté peut nécessiter des interventions de l'État pour lesquelles des sommes non négligeables sont prévues. Si ces mesures n’ont pas les effets escomptés, c'est qu'elles entrent en tension avec d'autres visant l'élévation des standards pour maintenir la compétitivité de la main-d'œuvre américaine dans un contexte de concurrence internationale.

Enfin, les principes libéraux font reposer sur les individus la responsabilité de construire les parcours qui prolongent la formation après le socle commun. Le nouveau modèle doit bien reconnaître que tous les individus ne disposent pas des mêmes ressources pour ce travail. Pour rester dans un cadre de justice, l'État managérial doit donc reprendre certaines orientations de l'État-providence. Comment aider les individus à faire le bilan de leurs compétences? À les confronter à la situation de l'emploi national et international? À identifier les ressources dont ils ont besoin? À se connecter aux bons réseaux? Surtout, comment protéger leur parcours: assurer leur revenu, leur protection sociale? Les partisans du nouvel ordre citent volontiers en exemple la flexicurité danoise. Ce compromis repose sur des traditions et des institutions propres aux pays scandinaves. À quelles conditions serait-il généralisable (Barbier, 2007)?

\section{RÉSUMÉ}

Le but de l'article est de déplacer les interrogations sur la crise du modèle de démocratisation en place depuis la fin de la Deuxième Guerre mondiale. Ce modèle est fondé sur une séparation de l'école et de la société. Ce qui était prôné comme une protection devient une perte de sens: les élèves d'origine populaire ne retrouvent pas leurs enjeux dans les «épreuves en papier» de l'école. Cette idée est illustrée par une analyse de la situation française et en particulier des résultats de la politique visant à amener $80 \%$ d'une génération au niveau du baccalauréat. La croissance des résultats a été spectaculaire de 1985 à 1995 . Depuis, la courbe flotte et tend même à décroître. Cette crise peut être interprétée dans un cadre plus large: le déclin de l'État-providence et la montée de l'État managérial. Celui-ci relève d'un nouveau modèle de démocratisation: plus de séparation entre le temps des études et le temps de la production, mais une formation tout au long de la vie; une gouvernance en réseau qui fait une large place aux intérêts économiques... Paradoxalement, ce nouveau modèle qui correspond à la nouvelle organisation du capitalisme pourrait peut-être répondre plus adéquatement au désengagement des classes populaires que l'ancienne forme scolaire.

\section{ABSTRACT}

The objective of this article is to refocus on the crisis of the democratization model set up after World War II. This model is based on the separation of education and society. What is acclaimed as a protection becomes a loss of meaning: students of working classes do not find their goals in the school 'examinations on paper'. This idea is illustrated by an analysis of the French situation and more specifically the results of the policy implemented to bring $80 \%$ of one generation to 
baccalaureate level. The improvement in the results rate was spectacular between 1985 and 1995 . Since then the curve has leveled off and even tends to decline. This crisis can be interpreted in a wider framework: the decline of the Welfare State and the expansion of the Manager State. This pertains to a new model of democratization: a greater separation between the time for study and time for production, but with more further education throughout the professional career, a networking authority giving much space to economic needs... Paradoxically this new model that corresponds to the new organization of capitalism may possibly respond more adequately to working classes defaulting than the old scholastic form.

\section{RESUMEN}

El objetivo del artículo es desplazar las interrogaciones sobre la crisis del modelo de democratización existente desde el final de la Segunda Guerra Mundial. Este modelo está basado en una separación de la escuela y la sociedad. Lo que se preconizaba como una protección se convierte en una pérdida de sentido: los alumnos de origen popular no encuentran lo que les interesa en los "exámenes escritos" de la escuela. Esta idea está ilustrada por un análisis de la situación francesa y en particular de los resultados de la política destinados a conducir un $80 \%$ de una generación al bachillerato. El crecimiento de los resultados fue espectacular de 1985 a 1995. Desde este tiempo, la curva fluctúa y tiende incluso a disminuir. Esta crisis puede interpretarse en un marco más amplio: la decadencia del Estado benefactor y la subida del Estado de gestión. Éste le sucede en el nuevo modelo de democratización: más separación entre el tiempo de los estudios y el tiempo de la producción, pero una formación a lo largo de la vida; una gobernanza en red que da más lugar a los intereses económicos... Paradójicamente, este nuevo modelo que corresponde a la nueva organización del capitalismo podría quizá responder más adecuadamente a la falta de compromiso de las clases populares que la antigua forma escolar.

\section{BIBLIOGRAPHIE}

Alain (1932). Propos sur l'éducation, Paris, PUF.

Ariès, P. (1975). L'enfant et la vie familiale sous l'Ancien Régime, Paris, Seuil.

BACKzo, B. (1982). Une éducation pour la démocratie: textes et projets de l'époque révolutionnaire, Paris, Garnier.

BALLION, R. (1994). Les lycéens et leurs petits boulots, Paris, Hachette.

BARBIER, J.C. (à paraître). "Pour une autre approche des différences culturelles: l'exemple du Danemark», in J.L. Derouet et M.C. Derouet-Besson (éd.), Repenser la justice dans le domaine de l'éducation et de la formation, Genève, Peter Lang.

Baudelot, C. et F. Leclercq (2005). Les effets de l'éducation, Paris, Documentation française.

Boltanski, L. et È. Chiapello (1999). Le nouvel esprit du capitalisme, Paris, Gallimard.

Chamboredon, J.C. (1988). «La sociologie de la socialisation: famille, école, agents d'encadrement et situations d'apprentissage», Revue Française de Pédagogie, n 83, p. 83-85.

Сharlot, B. (2001). Violences à l'école: état des savoirs, Paris, Bordas.

Crozier, M. (1987). État modeste, État moderne, Paris, Seuil.

DAlE, R. (2000). «Globalisation and Education: Demonstrating "A Common World Educational Culture" or Locating "A Globally Structured Educational Agenda" ", Educational Theory, vol. 50, n 4, p. 427-449.

DARRAS (1966). Le partage des bénéfices: expansion et inégalités en France, Paris, Minuit.

Darchy-Koechlin, B. et A. van Zanten (2005). «La formation des élites», Revue Internationale d'Éducation, $\mathrm{n}^{\circ} 39$. 
Dubet, F. (à paraître). "Les dilemmes de la justice», in J.L. Derouet et M.C. Derouet-Besson (éd.), Repenser la justice dans le domaine de l'éducation et de la formation, Genève, Peter Lang.

Durkheim, É. (1938). L'évolution pédagogique en France, Paris, PUF.

Duru-Bellat, M. (2006). L'inflation scolaire: les désillusions de la méritocratie, Paris, Seuil.

Finkielkraut, A. (1989). La défaite de la pensée, Paris, Gallimard.

Gewirtz, S. (à paraître). «Le Nouveau Parti travailliste et la Troisième Voie en éducation: tensions autour des Zones d'Action Éducative», in J.L. Derouet et M.C. Derouet-Besson (éd.), Repenser la justice dans le domaine de l'éducation et de la formation, Genève, Peter Lang.

Giddens, A. (1998). The Third Way: The Renewal of Social Democracy, London, Polity Press.

Hirschman, A.O. (1970). Exit, Voice and Loyalty: Responses to Decline in Firms, Organizations, and States, Cambridge, Harvard University Press.

Illich, I. (1970). Deschooling Society, New York, Harper \& Row.

Jellab, A. (2001). Scolarité et rapport aux savoirs en lycée professionnel, Paris, PUF.

Martinet, M. (1976). Culture prolétarienne, Paris, Maspero.

Maurin, É. (2007). La nouvelle question scolaire: les bénéfices de la démocratisation, Paris, Seuil.

Merle, P. (2002). La démocratisation de l'enseignement, Paris, La Découverte.

Nique, C. (2003). Comment l'école devint une affaire d'État: 1815-1840, Paris, Nathan.

OCDE (2004). Regards sur l'éducation: les indicateurs de l'OCDE, OCDE.

Plaisance, É (1985). L'échec scolaire, Paris, CNRS.

Prost, A. (1983). Les lycéens et leurs études à l'aube du XXI siècle, Paris, CNDP.

TANGuy, L. (2005). «De l'éducation à la formation: quelles réformes?», Éducation et Sociétés, Revue Internationale de Sociologie de l'Éducation, $n^{\circ} 16$.

van Haecht, A. (2004). «La posture critique en sociologie de l'éducation: tentatives d'état des lieux et de perspectives d'avenir», Éducation et Sociétés, Revue internationale de sociologie de l'éducation, $\mathrm{n}^{\circ} 13$, p. 5-10.

Verret, M. (1975). Le temps des études, Paris, Honoré Champion, 2 vol.

Vincent, G. (1980). L'école primaire française: une étude sociologique, Lyon, Presses Universitaires de Lyon. Willis, P. (1981). Learning to Labor: How Working Class Kids Get Working Class Jobs, New York, Columbia University Press. 